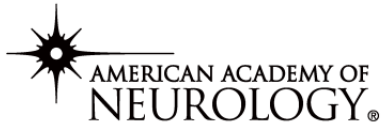

The most widely read and highly cited peer-reviewed neurology journal The Official Journal of the American Academy of Neurology

Neurology Publish Ahead of Print DOI: 10.1212/WNL.0000000000013225

\title{
Association of Fecal and Plasma Levels of Short-Chain Fatty Acids With Gut Microbiota and Clinical Severity in Parkinson Disease Patients
}

This is an open access article distributed under the terms of the Creative Commons AttributionNonCommercial-NoDerivatives License 4.0 (CC BY-NC-ND), which permits downloading and sharing the work provided it is properly cited. The work cannot be changed in any way or used commercially without permission from the journal.

Neurology® Published Ahead of Print articles have been peer reviewed and accepted for publication. This manuscript will be published in its final form after copyediting, page composition, and review of proofs. Errors that could affect the content may be corrected during these processes. 
Author(s):

Szu-Ju Chen, MD ${ }^{1,2,3}$; Chieh-Chang Chen, MD ${ }^{3,4}$; Hsin-Yu Liao, MSc ${ }^{5}$; Ya-Ting Lin, MSc ${ }^{5}$; Yu-Wei $\mathrm{Wu}, \mathrm{PhD}^{6}$; Jyh-Ming Liou, $\mathrm{MD}, \mathrm{PhD}^{4}$; Ming-Shiang $\mathrm{Wu}, \mathrm{MD}, \mathrm{PhD}^{4}$; Ching-Hua Kuo, $\mathrm{PhD}^{5,7,8}$; ChinHsien Lin, MD, PhD ${ }^{1}$

\section{Corresponding Author:}

Chin-Hsien Lin

q93421022@ntu.edu.tw

Affiliation Information for All Authors: 1. Department of Neurology, National Taiwan University Hospital, College of Medicine, National Taiwan University, Taipei, Taiwan; 2. Department of Neurology, National Taiwan University Hospital Bei-Hu Branch, Taipei, Taiwan;3. Graduate Institute of Clinical Medicine, College of Medicine, National Taiwan University, Taipei, Taiwan; 4. Division of Gastroenterology and Hepatology, Department of Internal Medicine, National Taiwan University Hospital, College of Medicine, National Taiwan University, Taipei, Taiwan;5. School of Pharmacy, College of Medicine, National Taiwan University, Taipei, Taiwan; 6. Graduate Institute of Biomedical Informatics, College of Medical Science and Technology, Taipei Medical University, Taipei, Taiwan; 7. The Metabolomics Core Laboratory, NTU Centers of Genomic and Precision Medicine, National Taiwan University, Taipei, Taiwan; 8. Department of Pharmacy, National Taiwan University Hospital, Taipei,

Taiwan

\section{Contributions:}

Szu-Ju Chen: Drafting/revision of the manuscript for content, including medical writing for content; Major role in the acquisition of data; Analysis or interpretation of data

Chieh-Chang Chen: Drafting/revision of the manuscript for content, including medical writing for content; Analysis or interpretation of data

Hsin-Yu Liao: Analysis or interpretation of data

Ya-Ting Lin: Analysis or interpretation of data

Yu-Wei Wu: Analysis or interpretation of data

Jyh-Ming Liou: Analysis or interpretation of data

Ming-Shiang Wu: Analysis or interpretation of data

Ching-Hua Kuo: Drafting/revision of the manuscript for content, including medical writing for content; Study concept or design; Analysis or interpretation of data

Chin-Hsien Lin: Drafting/revision of the manuscript for content, including medical writing for content; Major role in the acquisition of data; Study concept or design; Analysis or interpretation of data

Number of characters in title: 139

Abstract Word count: 350

Word count of main text: 4486

References: 50

Figures: 4

Tables: 3

Supplemental: Supplementary methods, 2 supplementary tables and 4 supplementary figures. Neurology checklist and STROBE checklist.

Statistical Analysis performed by: Szu-Ju Chen, MD, Department of Neurology, National Taiwan University Hospital, College of Medicine, National Taiwan University, Taipei, Taiwan. and Chin-Hsein Lin, MD, PhD. Department of Neurology, National Taiwan University Hospital, College of Medicine, National Taiwan University, Taipei, Taiwan.

Search Terms: [ 323 ] Class III, [ 149 ] Gastrointestinal, [ 165 ] Parkinson's disease/Parkinsonism 
Acknowledgements: The authors are grateful to the patients who participated in this study. We thank the staff of the Second and Eighth Core Lab, Department of Medical Research, National Taiwan University Hospital, for technical support during the study. We also thank the Ramaciotti Centre for Genomics, University of New South Wales (UNSW) for the shotgun sequencing in this study.

Study Funding: This work was supported by grants from National Taiwan University (NTU-110-ACC-5400-64841), National Taiwan University Hospital (NTUH 109-T18 and UN109-011), National Taiwan University Hospital Bei-Hu Branch (11005), and National Health Research Institutes (NHRIEX110-10716NC).

Disclosures: The authors report no disclosures relevant to the manuscript.

\section{Abstract}

Background and Objectives: Short-chain fatty acids (SCFAs) are gut microbial metabolites

that promote the disease process in a rodent model of Parkinson's disease (PD), but fecal

levels of SCFAs in PD patients are reduced. Simultaneous assessments of fecal and plasma

SCFA levels, and their inter-relationships with the PD disease process are scarce. We aimed

to compare fecal and plasma levels of different SCFAs subtypes in PD patients and healthy

controls to delineate their interrelations and link to gut microbiota changes and clinical

severity of PD.

Methods: A cohort of 96 PD patients and 85 controls were recruited from National Taiwan

University Hospital. Fecal and plasma concentrations of SCFAs were measured using

chromatography and mass spectrometry. Gut microbiota was analyzed using metagenomic

shotgun sequencing. Body mass index and medical co-morbidities were evaluated, and

dietary information was obtained using a food frequency questionnaire. To assess motor and

cognitive impairment, we used the Movement Disorder Society-Unified Parkinson's Disease 
Rating Scale (MDS-UPDRS) and the Mini-Mental Status Examination (MMSE).

Results: Compared with controls, PD patients had lower fecal but higher plasma concentrations of acetate, propionate, and butyrate. After adjustment for age, sex, disease duration, and anti-PD medication dosage, MDS-UPDRS part III motor scores correlated with reduced fecal levels of acetate $(\rho=-0.37, p=0.012)$, propionate $(\rho=-0.32, p=0.036)$, and butyrate $(\rho=-0.40, p=0.004)$ and with increased plasma propionate concentrations $(\rho=$ $0.26, p=0.042)$ in PD patients. MMSE scores negatively correlated with plasma levels of butyrate $(\rho=-0.09, p=0.027)$ and valerate $(\rho=-0.032, p=0.033)$ after adjustment for confounders. SCFAs-producing gut bacteria correlated positively with fecal levels of SCFAs in healthy controls but revealed no association in patients with PD. In the PD patient group, the abundance of pro-inflammatory microbes, such as Clostridiales bacterium NK3B98 and Ruminococcus sp. AM07-15, significantly correlated with decreased fecal levels and increased plasma levels of SCFAs, especially propionic acid.

Discussion: Reductions in fecal SCFAs but increased plasma SCFAs were observed in PD patients and corelated to specific gut microbiota changes and the clinical severity of PD.

\section{Classification of evidence:}

This study provides Class III evidence that gut metabolite SCFAs distinguish between PD patients and controls, and are associated with disease severity in patients with PD. 


\section{INTRODUCTION}

Emerging evidence suggests that the pathological hallmarks of PD, Lewy bodies,

originate in the gut enteric nervous system before the onset of motor dysfunction in patients

with PD. ${ }^{1}$ Studies in rodent PD models have shown that $\alpha$-synuclein aggregations could

propagate from the enteric nervous system to the brainstem through the vagus nerve. ${ }^{1,2}$

Gastrointestinal symptoms may precede motor symptoms by decades. ${ }^{3}$ This gut-brain

hypothesis has led to numerous studies showing that PD patients have a distinct gut

microbiota structure compared with controls and that changes in gut microbiota correlate with

disease severity and progression. ${ }^{4-6}$

Short-chain fatty acids (SCFAs) are the main metabolites produced by gut bacterial fermentation of dietary fiber and are speculated to be pivotal in gut-brain cross talks. ${ }^{7,8}$

SCFAs are absorbed in the colonocytes and transported to the liver via the portal venous

system, and some SCFAs enter the systemic circulation. Acetic acid (C2), propionic acid (C3)

and butyric acid (C4) are the most abundant SCFAs in the colon. ${ }^{9}$ SCFAs have local effects in

maintaining intestinal barrier integrity and shaping gut mucosal innate immunity. ${ }^{10}$ They also

have remote effects in the blood-brain barrier $(\mathrm{BBB})$ by promoting tight junction proteins

and crossing BBB to activate neurons through stimulation of $\mathrm{G}$ protein-coupled receptors. ${ }^{11,12}$

An in vivo study using a transgenic $\alpha$-synuclein-expressing mouse model demonstrated that a

germ-free environment eliminates disease phenotypes but that oral feeding with SCFAs re-

surfaced PD-related neuropathology by augmenting neuroinflammation. ${ }^{7}$ These findings

suggest that SCFAs are required for the development of PD process. However, results for 
SCFAs in PD patients conflict with these findings. Compared with healthy controls, PD

patients have reduced fecal SCFAs and their producing bacteria compared to healthy

controls. $^{8,13,14,15}$ Only a few studies have reported on circulating levels of SCFAs in patients

with PD. ${ }^{16,17}$ An integrated study examining both fecal and plasma levels of SCFAs is still

lacking. Hence, we aim to analyze and compare fecal and plasma levels of SCFAs in PD

patients and unaffected controls to delineate the interrelations of these levels and link to

changes in gut microbiota and clinical severity of PD.

\section{Primary research questions:}

Our primary research question was to determine whether fecal and plasma levels of SCFAs

could distinguish patients with PD from controls and correlate with disease severity in

patients with PD.

\section{METHODS}

\section{Standard protocol approvals, registrations, and patient consents}

The investigation conformed to the principles outlined in the Declaration of Helsinki. The study protocol was approved by the institutional review board of National Taiwan University Hospital. All participants signed written informed consent.

\section{Participants and clinical evaluation}

A total of 96 PD patients and 85 control participants were recruited from National Taiwan

University Hospital. PD was diagnosed according to the United Kingdom PD Society Brain 
Bank Clinical Diagnostic Criteria. ${ }^{18}$ Controls were neurologically unaffected participants who were spouses or accompanying friends of the PD patients. Participants were excluded if they had previously used neuroleptic agents; had a history of inflammatory bowel disorders, irritable bowel syndrome, colitis, colon cancer, use of antibiotics or probiotic supplements within 3 months of enrollment; or were vegetarians. Of the enrolled participants, $30 \mathrm{PD}$ patients and 24 controls had also received 16S ribosomal RNA sequencing, which was previously published. ${ }^{19}$

A comprehensive dietary history was collected using the Food Frequency Questionnaire (FFQ). ${ }^{20,21}$ We evaluated motor symptom severity with the Movement Disorder SocietyUnified Parkinson's Disease Rating Scale (MDS-UPDRS) part III score ${ }^{22}$ and Hoehn-Yahr staging during the "on" and "off" phase of PD. ${ }^{23}$ During "off" phase, patients with HoehnYahr stage $<3$ were classified as having early-stage PD and those with stage $\geq 3$ as having advanced-stage PD. Cognition was assessed with the Mini-Mental State Examination (MMSE) during the "on" phase of PD. ${ }^{24} \mathrm{PD}$ with dementia (PDD) was diagnosed according to the criteria proposed by the Movement Disorder Society. ${ }^{25}$ An MMSE score $\leq 25$ was applied as the cut-off value for significant cognitive impairment combined with any impairment in the eight instrumental activities for the diagnosis of PDD. ${ }^{26}$ The dosage of anti-PD medications during the study period was converted into a levodopa equivalent daily dosage (LEDD).

\section{Measurement of fecal and plasma levels of SCFAs}

We collected $10 \mathrm{~mL}$ of venous blood and fresh feces from each participant. Blood was 
sampled in the morning after participants had fasted for at least $10 \mathrm{~h}$. Total fecal DNA was

extracted using a QIAamp DNA Stool Mini Kit (Qiagen, Hilden, Germany). Fecal SCFA

analysis was performed using the fresh fecal sample collected in a plastic tube within 24

hours after collection. Dietary information was obtained on the same day of blood collection

using a self-administered FFQ. ${ }^{20,21}$ Fecal and plasma analyses for individual SCFAs were

performed with gas chromatography-mass spectrometry (GC-MS) and liquid chromatography

tandem MS (LC-MS/MS) as described in the eAppendix $1 .^{27,28}$

\section{Measurement of plasma cytokine levels}

The plasma levels of pro-inflammatory cytokines, including IL-2, tumor necrosis factor-

$\alpha($ TNF- $\alpha)$ and interferon- $\gamma($ IFN- $\gamma)$, were measured using the ProcartaPlex Human Th1/Th2

Cytokine Panel (Thermo Fisher Scientific, Vienna, Austria) according to the manufacturer's

instructions.

Gut microbiota analyses with shotgun metagenomic sequencing

Fecal samples were collected into stool specimen collection tubes containing DNA

stabilizer (Sarstedt), which were immediately flash-frozen on dry ice and stored at $-80^{\circ} \mathrm{C}$

before analyses. Total fecal DNA was extracted using a QIAamp DNA Stool Mini Kit

(Qiagen, Hilden, Germany), as previously described and detailed in eAppendix $1 .{ }^{19}$

\section{Statistical analysis}

Continuous variables are expressed as mean \pm standard deviation and categorical

variables as numbers and percentages. We tested the homogeneity of variances using

Levene's test. Variables were compared with two-tailed t-tests or analysis of variance 
(ANOVA) if normally distributed and with the non-parametric Mann-Whitney U test if assumptions of normality or homoscedasticity were violated. The concentrations of SCFAs in feces or plasma were log-transformed to achieve normal distribution before further analysis.

A logistic regression model was applied to examine the relationship between SCFA levels and the occurrence of PD, with adjustment for age and sex. We used the area under the receiver operating characteristic (ROC) curve (AUC) to quantify the model's diagnostic performance for exploring the ability of SCFAs to distinguish between PD patients and controls. To examine the associations between SCFAs and motor or cognitive symptom severity, we used multivariable linear regression models with MDS-UPDRS part III during "off” phase and MMSE scores as dependent factors. Independent variables included the log-transformed SCFAs levels, age, sex, disease duration, LEDD, and usage of entacapone. For shotgun sequencing and bioinformatic analysis, ${ }^{29,30}$ the details are addressed in the eAppendix 1.

\section{Data Availability}

The raw anonymized microbiome sequencing data were uploaded to the NCBI BioSample database (https://www.ncbi.nlm.nih.gov/biosample/) with the submission ID number SUB10308106. Anonymized data not published within this article will be made available by request from any qualified investigator.

\section{RESULTS}

A total of 181 participants including 96 PD patients $(67.5 \pm 11.5$ years old, $60.4 \%$ male $)$ and 85 normal controls $(64.8 \pm 8.2$ years old, $48.2 \%$ male $)$ were enrolled in this study. Of the 
enrolled PD patients, 10 patients were drug naïve (10.4\%), 12 patients had motor fluctuation

$(12.5 \%)$ and 9 patients experienced peak-dose dyskinesia (9.4\%) (Table 1). Among the control participants, $73(85.9 \%)$ were spouse controls. The dietary intake patterns in all participants in the past year using the FFQ are summarized in eTable 1,http://links.lww.com/WNL/B718. Patients and controls did not differ significantly in demographics or dietary intake patterns, including fiber intake, which is the main dietary source of SCFAs.

\section{Fecal and plasma levels of different types of SCFAs in PD patients and controls}

Patients with PD had significantly lower fecal concentrations of acetic acid $(p=0.004)$, propionic acid $(p=0.006)$, and butyric acid $(p=0.003)$ than controls (Table 1 and Figure 1AD). In contrast, plasma concentrations of circulating propionic acid $(p=0.009)$, butyric acid $(p=0.004)$, and valeric acid $(p=0.047)$ were increased in PD patients compared to controls

(Table 1 and Figure 2A-D).

We first examined the possible influence of age on fecal and plasma SCFAs levels. We found that age did not correlate with either measure for the individual SCFA types, except plasma levels of isovaleric acid modestly correlating with age in controls $(\rho=-0.37, p=$ 0.016). There were no significant correlations between fecal and plasma levels of the individual types of SCFAs (eFigure 1,http://links.lww.com/WNL/B718). We further observed that plasma levels of acetic acids correlated with $\operatorname{IFN}-\gamma(\rho=0.301, p=0.003)$ and showed a trend with TNF- $\alpha(\rho=0.197, p=0.051)$; while plasma levels of butyric acids tended to correlate with IFN- $\gamma(\rho=0.194, p=0.06)$ (eFigure 2,http://links.lww.com/WNL/B718). 
We next examined whether fecal or plasma levels of individual SCFA types distinguished between PD patients and controls. With adjustment for age and sex in multivariate logistic regression analysis, higher fecal levels of acetic acid (odds ratio [OR] 0.16, 95\% confidence interval [CI], 0.04-0.57, $p=0.006$ ), propionic acid (OR 0.20, 95\% CI, 0.06-0.60, $p=0.006$ ), and butyric acid (OR 0.27, 95\% CI, 0.10-0.67, $p=0.007)$ were associated with a lower chance to be patients with PD. In contrast, the occurrence of PD was associated with higher plasma levels of propionic acid (OR 14.4, 95\% CI 1.63-159, $p=0.021)$, butyric acid (OR 17.8, 95\% CI 2.50-152, $p=0.006)$, and valeric acid (OR 8.70, 95\% CI 1.49-67.3, $p=0.025)$ (eTable2,http://links.lww.com/WNL/B718).

Based on the results of the logistic regression analyses, we next performed ROC curve analysis to predict PD occurrence. The prediction accuracy, expressed as the AUC, improved from 0.55 in the basic model (age and sex, $p=0.357$ ) to 0.65 with the addition of fecal levels of acetic acid, propionic acid, and butyric acid, which differed significantly between the PD and control groups in the multivariate logistic regression analysis $(p=0.004)$. The AUC was 0.67 with the addition of plasma levels of propionic acid, butyric acid, and valeric acid ( $p=$ 0.003), which differed significantly between PD patients and controls in the multivariate logistic regression analysis. The AUC further improved to $0.72(p<0.001)$ in the full model, which included age, sex, and the fecal and plasma levels of the targeted SCFAs (Figure 3A and Table 2).

\section{Fecal and plasma SCFAs levels and motor symptom severity}

Fecal levels of acetic acid, propionic acid, butyric acid, and valeric acid were all 
significantly reduced in patients with advanced-stage PD compared to those with early-stage disease (all $p<0.01$; eFigure 3A,http://links.lww.com/WNL/B718). In contrast, plasma levels of valeric acid were significantly increased in patients with advanced-stage disease compared to those with early-stage PD (advanced-stage vs. early-stage: $0.18 \pm 0.19$ vs. $0.06 \pm 0.06 \mu \mathrm{M}$, $p=0.003$; eFigure 3B). Furthermore, fecal levels of acetic acid $(\rho=-0.37, p=0.005)$, propionic acid $(\rho=-0.32, p=0.015)$, and butyric acid $(\rho=-0.40, p=0.002)$ negatively correlated with MDS-UPDRS part III motor scores (Figure 3B-D). However, plasma levels of propionic acid trended to correlate with MDS-UPDRS part III scores $(\rho=0.26, p=0.086$, eFigure 3C,http://links.lww.com/WNL/B718).

After adjustment for age, sex, disease duration, LEDD, and entacapone use, multivariate linear regression analysis revealed negative correlations of MDS-UPDRS part III scores with fecal levels of acetic acid, propionic acid, butyric acid, and isobutyric acid (Table 3). We included adjustment for LEDD and entacapone use in the multivariate analyses because of a recent study reporting that circulating propionic acid level is negatively correlated with entacapone use. ${ }^{15}$ Plasma propionic acid levels positively correlated with MDS-UPDRS part III scores after adjustment for the covariates (coefficient $=24.4,95 \%$ CI $1.00-47.7, p=$ 0.042; Table 3). These findings suggested that lower fecal levels of most SCFAs and increased plasma levels of propionic acid were associated with worse motor performance in PD patients.

\section{Fecal and plasma SCFAs levels and cognitive symptom severity}

Fecal levels of different types of SCFAs were comparable between PD patients with 
normal cognition and those with PDD (eFigure 4A,http://links.lww.com/WNL/B718).

However, plasma levels of isobutyric acid $(0.37 \pm 0.17$ vs. $0.26 \pm 0.08 \mu \mathrm{M}, p=0.023)$ and valeric acid $(0.19 \pm 0.21$ vs. $0.07 \pm 0.06 \mu \mathrm{M}, p=0.005)$ were significantly higher in PDD patients than in PD patients with normal cognition (eFigure 4B,http://links.lww.com/WNL/B718). Consistent with this pattern, plasma levels of isobutyric acid $(\rho=-0.41, p=0.014)$ correlated negatively with MMSE scores, and valeric acid levels showed a trend to negatively correlate with MMSE scores in PD patients ( $\rho=$ $0.32, p=0.070 ;$ eFigure 4C,http://links.lww.com/WNL/B718).

With adjustment for age, sex, disease duration, LEDD, and entacapone use in the multivariate linear regression model, plasma levels of butyric acid (coefficient $=-11.7,95 \%$ CI -21.9 to $-1.44, p=0.027$ ) and valeric acid (coefficient $=-6.58,95 \%$ CI -12.6 to $-0.60, p=$ 0.033) were persistently found to negatively correlate with MMSE scores (Table 3), whereas fecal levels of butyric acid positively correlated with MMSE scores (coefficient $=2.95,95 \%$ CI $0.60-5.31, p=0.015$; Table 3). These findings suggested that lower fecal but higher plasma butyric acid and increased plasma valeric acid were associated with a worse cognition in $\mathrm{PD}$ patients.

\section{Gut microbiome species correlate with plasma and fecal levels of SCFAs}

Metagenomic analysis of gut microbiome species showed that the bacterial richness within each sample ( $\alpha$-diversity) tended to be higher in PD patients than in healthy controls (eFigure 5A,http://links.lww.com/WNL/B718), which are consistent with previous studies. ${ }^{19}$ The principal coordinates analysis revealed that PD patients had different gut microbiota 
composition from healthy controls ( $B$-diversity, analysis of similarities, $p=0.05$; eFigure

5B,http://links.lww.com/WNL/B718).

We further explored the correlations between the relative abundance of gut microbiota and fecal or plasma levels of SCFAs. Heat maps representing Spearman correlations for the relative abundance of differential bacteria and SCFA concentrations in feces or plasma revealed that fecal levels of acetic acid, propionic acid, and butyric acid all correlated with the abundance of Bacteroides sp. AM16-15 and Bacteroides sp. AM25-34, in control participants (eFigure 6A,http://links.lww.com/WNL/B718). However, we did not find these positive correlations in patients with PD (eFigure 6B,http://links.lww.com/WNL/B718). Among PD patients, the abundance of pro-inflammatory microbes, such as Clostridiales bacterium NK3B98 and Ruminococcus sp. AM07-15, significantly correlated with decreased fecal levels and increased plasma levels of SCFAs, especially propionic acid (eFigure 6B, C,http://links.lww.com/WNL/B718). Of note, plasma propionic acid levels were associated with PD occurrence and with motor symptom severity (Table 2 and 3). Furthermore, the abundance of Ruminococcus sp. AM28-29LB consistently correlated with reduced fecal levels of all SCFAs and increased plasma butyric acid (eFigure 6B, C,http://links.lww.com/WNL/B718), which was associated with cognitive decline in PD (Table 3).

While combing the fecal and plasma SCFAs data as plasma-to-fecal ratios of the individual types of SCFAs, the abundance of Clostridiaceae bacterium AF31-3BH correlated with an elevated plasma-to-fecal ratio of propionic acid (Figure 4), a species closely related 
to Clostridium bacterium. The abundance of Roseburia sp. Am16-25 correlated with the

plasma-to-fecal ratio of acetic acid, and the abundance of Bifidobacterium bifidum and

Eisenbergiella massiliensis correlated with an increased plasma-to-fecal ratio of butyric acid,

which also was increased in patients with PDD. Of note, the abundance of Prevotella sp. P3-

122 was negatively associated with the plasma-to-fecal ratios of all measured SCFAs (Figure

4). Furthermore, as shotgun metagenomics analysis could provide extensive metabolic

prediction capabilities, we observed that plasma levels of SCFAs, especially propionic acid,

positively correlated with pathways of peptidoglycan maturation, guanosine nucleotides

biosynthesis, L-aspartate and L-asparagine biosynthesis, and glycolysis IV (eFigure

7,http://links.lww.com/WNL/B718). Taken together, these findings indicate that alterations in the abundance of specific gut microbiota species with related metabolic pathways in PD

patients relative to unaffected controls are associated with plasma and fecal levels of different

SCFAs, which correlate with either motor or cognitive severity in PD.

\section{Classification of evidence:}

This study provides Class III evidence that gut metabolite SCFAs distinguish between PD

patients and controls, and are associated with disease severity in patients with PD.

\section{Discussion}

To the best of our knowledge, this study is the first integrated study concomitantly

examining both plasma and fecal levels of individual SCFAs types and linking to changes in gut microbiota and symptom severity in patients with PD compared with unaffected controls. 
We found that PD patients had lower fecal but higher plasma concentrations of SCFAs compared to controls. We further demonstrated that after adjustment for confounders, the reduced fecal levels but increased plasma levels of SCFAs associated with gut microbiota changes and the clinical severity of PD.

In stool, we observed lower levels of SCFAs in PD patients than controls, in line with several studies that previously founded reduced fecal SCFAs levels in PD patients. ${ }^{8,13,14,15}$ Similar to the findings of the current study, Tan et al also found an association between reduced fecal SCFAs and worse cognitive and motor outcomes; ${ }^{14}$ while Aho et al. did not find correlations between fecal SCFAs with non-motor symptom burden in PD. ${ }^{15}$ Consistently, recent evidence indicates that SCFAs-producing microbiota are also less abundant in association with PD disease severity, which predicts a rapid motor and cognitive deterioration. ${ }^{43}$ In addition, we found that reduced fecal levels of SCFAs could distinguish PD patients from controls and extend the current knowledge that levels of these SCFAs negatively correlated with motor severity after adjustment for potential confounders. Moreover, cognitive decline is one of the most disturbing non-motor symptoms of PD. We observed that reduced fecal levels of butyric acid also correlated with cognitive decline in patients with PD. Gut luminal SCFAs are absorbed in colonocytes and are beneficial for maintaining the intestinal barrier and homeostasis. ${ }^{34}$ Reduced fecal SCFAs, especially butyric acid, are linked to the loss of intestinal tight junctions leading to leaky gut and increased intestinal inflammation, which is associated with aggravating the PD disease process. ${ }^{34}$ In healthy controls of this study, we found a positive correlation of fecal acetic acid, propionic 
acid, and butyric acid levels with the abundance of Bacteroides sp. AM16-15 and Bacteroides sp. AM25-34, which are SCFA-producing microbiota. ${ }^{32,35}$ Notably, this correlation was not present in patients with PD. In line with these findings, in a recent study in a European population, a positive correlation between SCFAs and relative abundance of Bacteroides genera was reported in controls but not in PD patients. ${ }^{15}$ Our results support one previous study, which found SCFAs-producing bacteria to be reduced in PD but not patients with rapid eye movement sleep behavior disorder, suggesting that SCFAs may play a role in the development of PD. ${ }^{36}$

In plasma, we identified contradictory results that PD patients had higher plasma concentrations of SCFAs than controls, except acetic acid. Plasma levels of propionic acid correlated with motor symptom severity, whereas butyrate and valerate concentrations associated with cognitive decline. One recent study similarly found plasma propionate level to be higher in PD patients than controls; while the plasma levels of acetic acid and propionic acid decreased in patients with multiple system atrophy compared to those with PD. ${ }^{17}$ A casecontrol study in a Korean population also showed that plasma acetic acid was increased in PD patients even though the level did not correlate with motor symptom severity. ${ }^{16}$ Recent evidence also revealed that the concentrations of SCFAs are elevated in saliva and urine in PD patients compared to the general population. ${ }^{15,37}$ Most of the SCFAs produced in the colon are absorbed and utilized by colonocytes leaving only a minority entering the peripheral circulation. ${ }^{12}$ The current findings demonstrate that systemic levels of SCFAs were increased even as fecal levels of SCFAs were consistently reduced in patients with PD. One 
possible explanation for this divergence may come from increased intestinal permeability that allows SCFAs to enter the systemic circulation, implying an intestinal barrier malfunction. In support of this hypothesis, increased fecal zonulin level, a marker of gut permeability, and increased fecal calprotectin, a marker of gut inflammation, were recently reported in PD patients compared to controls. ${ }^{15}$ In vivo rodent model studies also have shown that one of the earliest pathological changes in PD is intestinal inflammation with impaired intestinal tight junctions that allow for increased infiltration of gut microbial metabolites into the systemic circulation. ${ }^{38}$ The "leaky gut phenomenon" in patients with PD may lead to a higher penetration of SCFAs from gut to the systemic circulation. However, among the subtypes of SCFAs, plasma level of acetic acid was not significantly increased in PD patients. In addition to the possible "leaky gut phenomenon" effect, several conditions may contribute to the plasma levels of acetic acid. Acetic acid can be endogenously produced and the plasma level is influenced by metabolic stress, such as prolonged fasting, lipolysis and glucagon-like peptide-1concentrations. ${ }^{39}$ These factors may influence the observed comparable plasma levels of acetic acid between PD patients and controls in this study. The elevated SCFAs in systemic circulation may cross the blood-brain barrier and impact the neuron-microglia microenvironment in the central nervous system. Although accumulating evidence support the beneficial effects of SCFAs, including maintaining BBB integrity and affecting neurogenesis through epigenetic mechanisms, SCFAs may also exert pathological effects if the level is over a concentration range which was shown to promote neuroinflammation in a transgenic $\alpha$-synuclein rodent model. ${ }^{7}$ Further studies on the molecular mechanisms of 
different SCFAs at various concentration range in the central nervous system are warranted.

Furthermore, while motor symptom severity positively correlated with plasma propionic acid

levels, cognitive dysfunction correlated with circulating butyric acid and valeric acid

concentrations. These associations may reflect the diverse pathophysiological processes of

motor and cognitive decline in PD. ${ }^{37}$ Links between systemic levels of valeric acid and brain

pathology also have been reported in patients with Alzheimer's disease,${ }^{40}$ with higher plasma

valeric acid associated with a greater degree of amyloid deposition and circulating

inflammatory marker expression. In addition, levels of propionic acid in the systemic

circulation correlated with motor symptom severity in the current work. In earlier studies,

increased levels of this SCFA were reported to trigger mitochondrial dysfunction, activate T

cell immunity, and increase oxidative stress, especially in the striatum, leading to motor

dysfunction in patients with autism spectrum disorder. ${ }^{41}$ The positive correlations between

plasma levels of SCFAs and inflammatory markers in this study could be interpreted as

supporting a relevant association between SCFAs and systemic inflammation. Further

experimental studies exploring the molecular mechanisms of individual types of SCFAs in

promoting neuroinflammation and neurodegeneration are warranted.

The plasma-to-fecal ratio of individual types of SCFAs could provide a better landscape of

individual SCFAs in the leaky gut condition of PD process. We observed that the plasma-to-

fecal ratios of separated SCFAs types were linked to the respective abundance of specific gut

microbiota. For example, Bifidobacteria and Bacteroides spp. produce acetate, whereas

microbes from the Firmicutes phylum (Roseburia, Eubacterium, and Lachnospira) actively 
produce butyrate. ${ }^{42}$ The Bacteroides spp. are Gram-negative Bacteroidetes and predominate in the human gut. Acetic acid is the most abundant SCFA and acts in the maintenance of the normal structure, integrity, and function of the intestines. ${ }^{34}$ The absence of positive correlations between Bacteroides spp. abundance and fecal levels of all types of SCFAs in patients with PD may suggest a dysbiosis state that leads to impaired intestinal barrier function in the disease process of PD. ${ }^{34}$ In addition, among PD patients, we found that the abundance of the pro-inflammatory Clostridiales bacterium correlated with the plasma-tofecal ratio of propionic acid, whereas the abundance of another pro-inflammatory microbe, Ruminococcus sp. AM28-29B, correlated with an increased plasma level of butyric acid.

Clostridiales bacterium and Clostridiaceae taxa, such as Clostridium spp., are also associated with inflammatory bowel diseases. ${ }^{43}$ The abundance of members of the Clostridiales order correlates positively with the regulatory T-cell transcription factor Foxp3, as does abundance of members of the phylum Verrucomicrobia. ${ }^{44}$ We therefore speculate that Clostridiales bacterium and Ruminococcus sp. AM28-29B may be able to induce intestinal regulatory immune responses leading to intestinal inflammation and possibly explain the increased plasma-to-fecal ratios of propionic acid and butyric acid. Of note, the abundance of Prevotella spp. P3-122 correlated with reduced plasma-to-fecal ratios of all SCFAs. Several studies, including our previous work, have shown a decreased abundance of Prevotellaceae and Prevotella genera in patients with PD. ${ }^{6,19}$ As commensals, Prevotellaceae bacteria are involved in mucin synthesis in the gut mucosal layer and in production of neuroactive SCFAs through fiber fermentation. ${ }^{45}$ The Prevotella sp. P3-122 may partially explain the decreased 
plasma-to-fecal ratio of all SCFAs in patients with PD. Finally, although numerous studies

have shown that propionic acid and butyric acid have anti-inflammatory effects, results conflict. ${ }^{7,13}$ Both pro- and anti-inflammatory activities of SCFAs have been documented, and dietary supplementation with SCFAs produces variable effects on inflammation that appear to be strongly influenced by SCFA concentration. Our further analysis showed plasma levels of SCFAs, especially propionic acids, positively correlated with peptidoglycan maturation pathway and glycolysis IV. Peptidoglycan is one of the pathogen-associated molecular patterns and has been shown to enhance proinflammatory cytokine secretion from activated microglia. ${ }^{46}$ Glycolysis is the conversion of glucose to pyruvate or lactate which results in the generation of ATP and has been shown to be abnormally regulated in neurons in patients with PD. ${ }^{47}$ An in vivo model of zebrafish deficient in the familial PD associated protein PTENinduced kinase 1 observed an upregulation of TigarB, the ortholog of human TP53 Induced Glycolysis Regulatory Phosphatase (TIGAR). ${ }^{48}$ Further investigation is needed into how different concentrations of individual SCFAs types affect immune responses in the context of PD pathology.

Our study has several limitations. First, we did not directly measure colonic SCFA concentrations but used fecal SCFA levels as a surrogate for colonic SCFA production and absorption. Second, we did not examine intestinal barrier function using a gut permeability assay in our participants, although one recent study has demonstrated gut hyper-permeability in PD patients compared to controls. ${ }^{15}$ Third, cognitive function was assessed using MMSE. Further detailed neuropsychological tests are needed for assessing the correlation between 
SCFAs levels and individual cognitive domain decline in patients with PD. Fourth, dietary

intake pattern assessed by FFQ was comparable between PD patients and controls, while some other studies reported between-group differences. ${ }^{49,50}$ As the some controls enrolled in this study were spouses of the PD patients, we speculate the common environment shared between spouse controls and PD patients may contribute to the comparable dietary intake pattern between PD patients and controls in this study. In addition, constipation and use of laxatives and anti-cholinergic agents or cholinesterase inhibitors may influence the results of gut microbiota and gut metabolites. Studies enrolling healthy controls from general populations and considering these potential confounders should be taken into account in the future. Furthermore, the diagnosis of PD was based on clinical diagnostic criteria in this study and lacks neuropathology confirmation. Finally, the cross-sectional study design precludes any inferences of a causal relationship between SCFAs and the disease process in PD. Further longitudinal follow-up studies with serial measurements of both fecal and plasma SCFAs are warranted.

In conclusion, our findings suggest that gut metabolite SCFAs may serve as surrogate gut-oriented markers for distinguishing PD patients from controls and reflecting disease severity. Further studies of the exact mechanisms of different SCFAs at neurons and microglia in experimental models should help to elucidate the complex gut-brain interactions in PD. 


\section{Figure legends}

Figure 1. Comparison of the fecal levels of SCFAs in PD patients and healthy controls.

Violin plot showing data density and median with interquartile range (solid and dashed

horizontal lines) for fecal levels of acetic acid (A), propionic acid (B), butyric acid (C) and

valeric acid (D) in PD patients and unaffected controls. The mean \pm standard deviation (solid

horizontal line, dashed horizontal line) is shown. $* p<0.05, * * p<0.01$. PD, Parkinson's

disease.
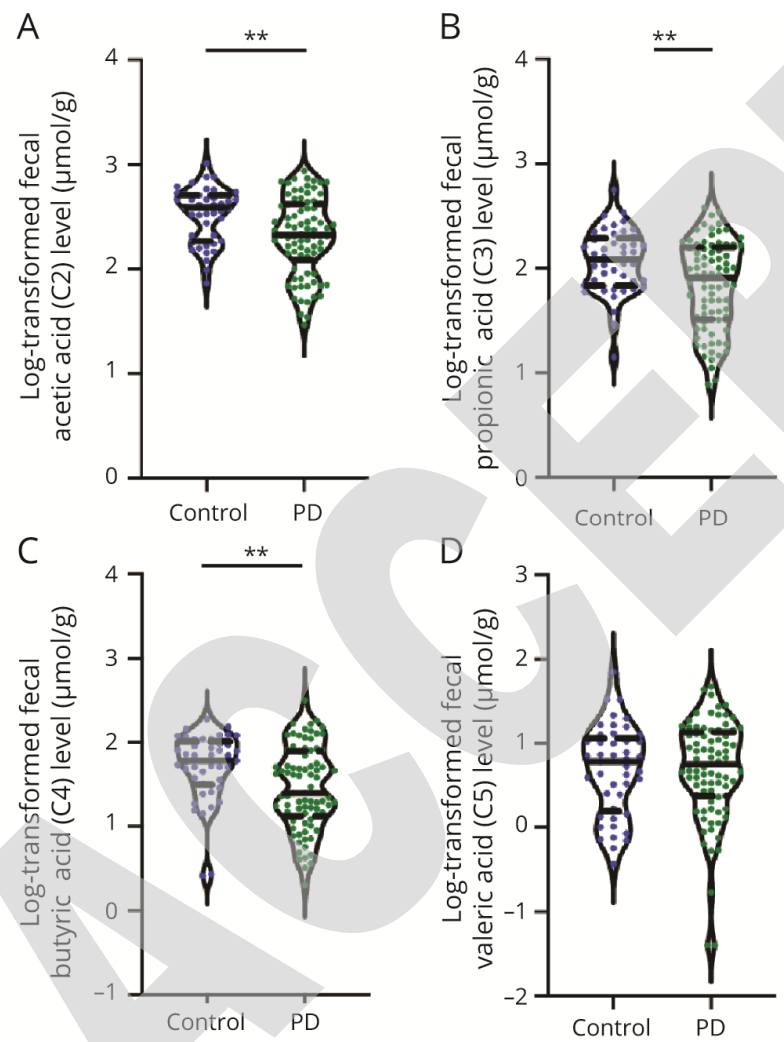


\section{Figure 2. Comparison of the plasma concentrations of SCFAs in PD patients and}

healthy controls. Violin plot showing data density and median with interquartile range (solid and dashed horizontal lines) for plasma concentrations of acetic acid (A), propionic acid (B), butyric acid (C) and valeric acid (D) in PD patients and unaffected controls. The mean \pm standard deviation (solid horizontal line, dashed horizontal line) is shown. * $p<0.05$, ** $p<$ 0.01. PD, Parkinson's disease.

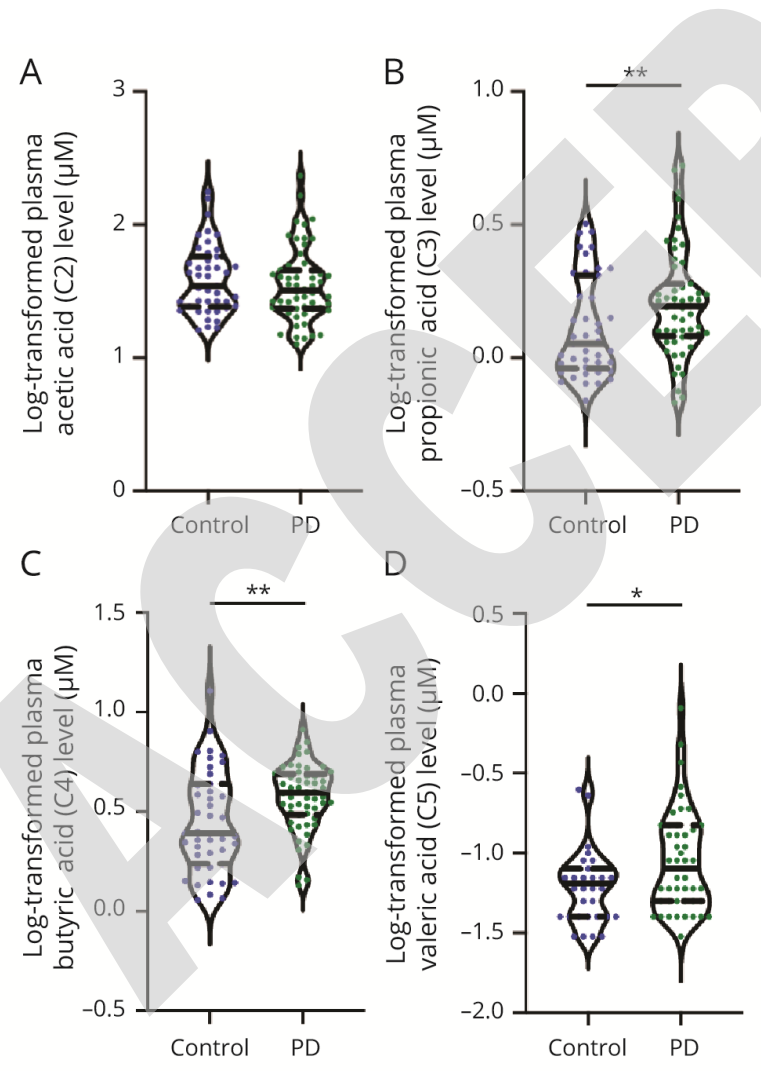


Figure 3. ROC curves for distinguishing PD patients from controls in participants, and correlations between SCFAs levels and clinical motor symptom severity in patients with

PD.

(A) The accuracy of estimating PD using age and sex alone $(\mathrm{AUC}=0.55, p=0.357)$

significantly improved with the addition of fecal levels of acetic acid, propionic acid, and butyric acid, which showed differences between PD patients and controls $(\mathrm{AUC}=0.65, p=$

0.004). The accuracy of estimating the occurrence of PD further improved in a full model incorporating both fecal and plasma levels of targeted SCFAs, which showed differences between PD and controls $(\mathrm{AUC}=0.72, p<0.001)$. (B-D) Scatter plots show negative correlations between MDS-UPDRS part III motor scores and fecal levels of acetic acid (B), propionic acid (C), and butyric acid (D). MDS-UPDRS, Movement Disorder Society-Unified Parkinson's Disease Rating Scale; SCFAs, short-chain fatty acids; AUC, area under the curve; ROC, receiving operating characteristic. 

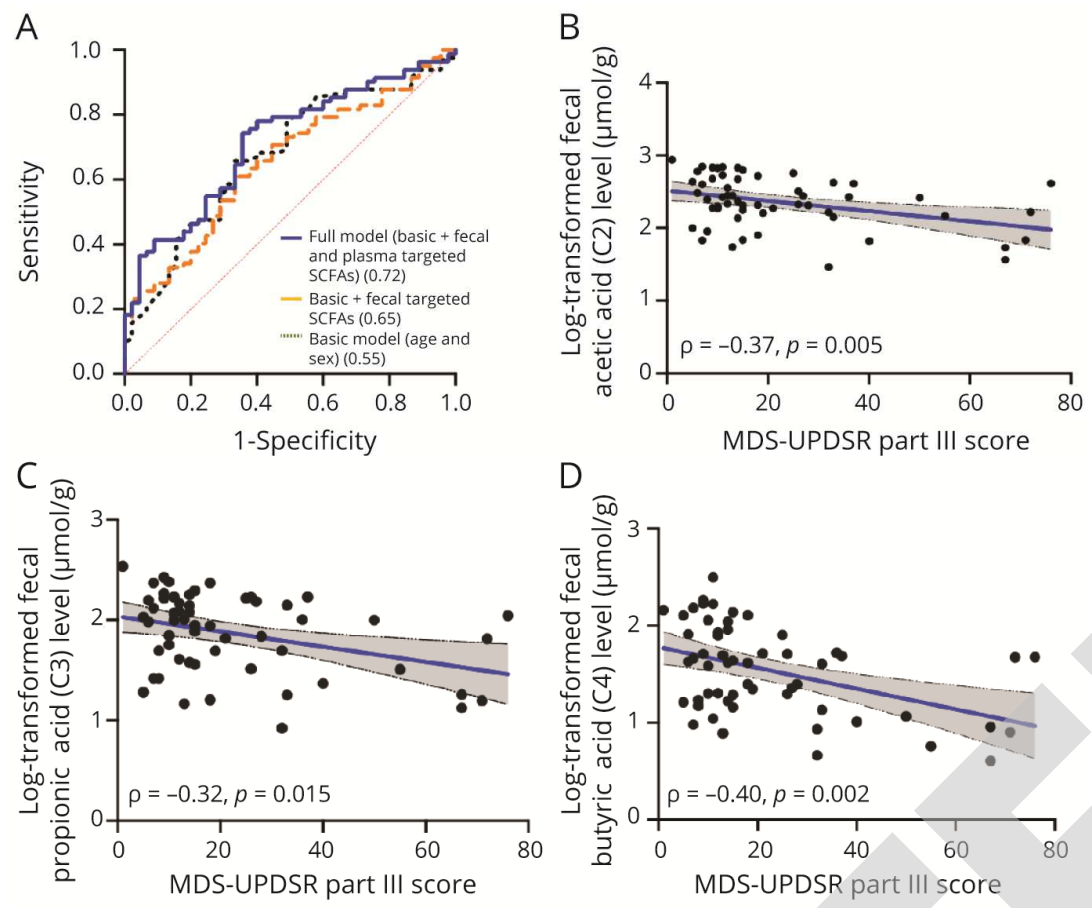
Figure 4. Heat maps representing the Spearman correlation of the relative abundance of

different bacteria and the plasma-to-fecal ratios of SCFAs. Correlations between plasma-

to-fecal ratios of different types of SCFAs in patients with PD. The $r$ values are represented

by gradient colors, where red and blue cells indicate positive and negative correlations,

respectively. Significant differences were indicated by $* p<0.05$ and $* * p<0.01$,

respectively. PD, Parkinson's disease; SCFAs, short-chain fatty acids.

Clostridiaceae bacterium AF31-3BH Clostridium sp. AM49-4BH

Alistipes shahi Blautia schinki Alistipes sp. AF14-19

Anaerostipes hadrus

Alistipes onderdonki

Neobitarella massiliensis

Anaerofilum sp. An201

Clostridium phoceensis

Bifidobacterium bifidum

Parabacteroides sp. AF27-14

Ruthenibacterium lactiformans

Roseburia sp. AM51-8

Roseburia sp. AM16-25

Eisenbergiella massiliensis

Prevotella sp. P3-122

Clostridium sp. AM25-23AC

Lachnospiraceae bacterium 1_4_56FAA

[Clostridium] glycyrrhizinilyticum

Bacteroides sp. AM22-3LB

Bacteroides massiliensis

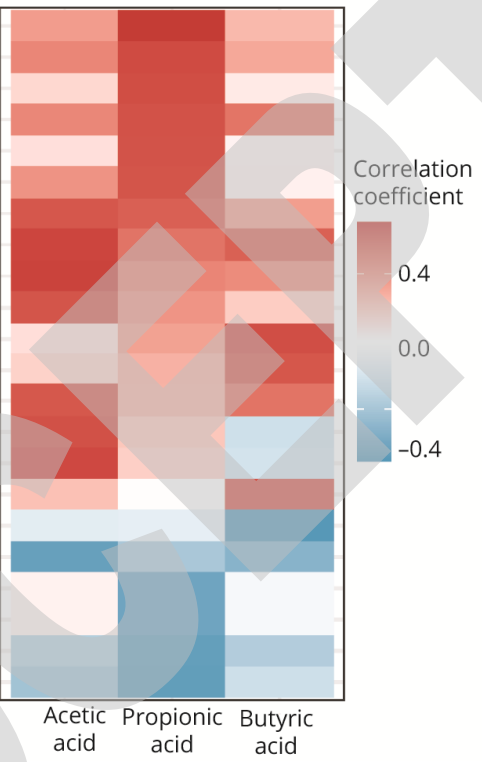




\section{Supplement-http://links.lww.com/WNL/B718}

\section{References}

1. Klingelhoefer L, Reichmann H. Pathogenesis of Parkinson disease-the gut-brain axis and environmental factors. Nat Rev Neurol. 2015;11(11):625-636; doi:

10.1038/nrneurol.2015.197.

2. Kim S, Kwon SH, Kam TI, et al. Transneuronal Propagation of Pathologic $\alpha$-Synuclein from the Gut to the Brain Models Parkinson's Disease. Neuron. 2019;103(4):627-641; doi: 10.1016/j.neuron.2019.05.035.

3. Schrag A, Horsfall L, Walters K, Noyce A, Petersen I. Prediagnostic presentations of Parkinson's disease in primary care: a case-control study. Lancet Neurol. 2015;14(1):57-64; doi:10.1016/S1474-4422(14)70287-X.

4. Nishiwaki H, Ito M, Ishida T, et al. Meta-Analysis of Gut Dysbiosis in Parkinson's

Disease. Mov Disord. 2020;35(9):1626-1635; doi: 10.1002/mds.28119.

5. Qian Y, Yang X, Xu S, et al. Gut metagenomics-derived genes as potential biomarkers of Parkinson's disease. Brain. 2020;143(8):2474-2489; doi: 10.1093/brain/awaa201.

6. Scheperjans F, Aho V, Pereira PA, et al. Gut microbiota are related to Parkinson's disease and clinical phenotype. Mov Disord. 2015;30 (3):350-358; doi: 10.1002/mds.26069.

7. Sampson TR, Debelius JW, Thron T, et al. Gut Microbiota Regulate Motor Deficits and Neuroinflammation in a Model of Parkinson's Disease. Cell. 2016;

167(6):1469-1480; doi: 10.1016/j.cell.2016.11.018. 
8. Unger MM, Spiegel J, Dillmann KU, et al. Short chain fatty acids and gut microbiota differ between patients with Parkinson's disease and age-matched controls. Parkinsonism Relat Disord. 2016;32:66-72; doi: 10.1016/j.parkreldis.2016.08.019.

9. Cummings JH, Pomare EW, Branch WJ, Naylor CP, Macfarlane GT. Short chain fatty acids in human large intestine, portal, hepatic and venous blood. Gut. 1987;28(10):12211227; doi: 10.1136/gut.28.10.1221.

10. Blacher E, Levy M, Tatirovsky E, Elinav E. Microbiome-Modulated Metabolites at the Interface of Host Immunity. J Immunol. 2017;198(2):572-580; doi:

10.4049/jimmunol.1601247.

11. Vijay N, Morris ME. Role of monocarboxylate transporters in drug delivery to the brain.

Curr Pharm Des. 2014;20 (10):1487-1498; doi: 10.2174/13816128113199990462.

12. Dalile B, Van Oudenhove L, Vervliet B, Verbeke K. The role of short-chain fatty acids in microbiota-gut-brain communication. Nat Rev Gastroenterol Hepatol.

2019;16 (8):461-478; doi: 10.1038/s41575-019-0157-3.

13. Mulak A. A controversy on the role of short-chain fatty acids in the pathogenesis of Parkinson's disease. Mov Disord. 2018;33 (3):398-401; doi: 10.1002/mds.27304.

14. Tan AH, Chong CW, Lim SY, et al. Gut Microbial Ecosystem in Parkinson Disease: New Clinicobiological Insights from Multi-Omics. Ann Neurol. 2021;89(3):546-559; doi: 10.1002/ana.25982.

15. Aho VTE, Houser MC, Pereira PAB, et al. Relationships of gut microbiota, short-chain fatty acids, inflammation, and the gut barrier in Parkinson's disease. Mol Neurodegener. 
2021;16(1):6; doi: 10.1186/s13024-021-00427-6.

16. Shin C, Lim Y, Lim H, Ahn TB. Plasma Short-Chain Fatty Acids in Patients With

Parkinson's Disease. Mov Disord. 2020;35(6):1021-1027; doi: 10.1002/mds.28016.

17. He X, Qian Y, Xu S, et al. Plasma Short-Chain Fatty Acids Differences in Multiple

System Atrophy from Parkinson's Disease. J Parkinsons Dis. 2021;11(3):1167-1176; doi:

10.3233/JPD-212604.

18. Hughes AJ, Daniel SE, Kilford L, Lees AJ. Accuracy of clinical diagnosis of idiopathic

Parkinson's disease: a clinico-pathological study of 100 cases. J Neurol Neurosurg

Psychiatry. 1992;55 (3):181-184; doi: 10.1136/jnnp.55.3.181.

19. Lin $\mathrm{CH}$, Chen $\mathrm{CC}$, Chiang HL, et al. Altered gut microbiota and inflammatory cytokine responses in patients with Parkinson's disease. J Neuroinflammation. 2019;16(1):129; doi:

10.1186/s12974-019-1528-y.

20. Chiu TH, Huang HY, Chen KJ, et al. Relative validity and reproducibility of a quantitative FFQ for assessing nutrient intakes of vegetarians in Taiwan. Public Health Nutr. 2014;17 (7):1459-1466; doi: 10.1017/S1368980013001560.

21. Chiu THT, Chang HR, Wang LY, Chang CC, Lin MN, Lin CL. Vegetarian diet and incidence of total, ischemic, and hemorrhagic stroke in 2 cohorts in Taiwan. Neurology 2020;94(11):e1112-e1121; doi: 10.1212/WNL.0000000000009093.

22. Goetz CG, Tilley BC, Shaftman SR, et al. Movement Disorder Society-sponsored revision of the Unified Parkinson's Disease Rating Scale (MDS-UPDRS): scale presentation and clinimetric testing results. Mov Disord. 2008;23(15):2129-2170; doi: 
$10.1002 / \mathrm{mds} .22340$.

23. Hoehn MM, Yahr MD. Parkinsonism: onset, progression and mortality. Neurology.

1967;17 (5):427-442; doi: 10.1212/wnl.17.5.427.

24. Folstein MF, Folstein SE, McHugh PR. "Mini-mental state". A practical method for grading the cognitive state of patients for the clinician. J Psychiatr Res. 1975;12(3):189-198;

doi: 10.1016/0022-3956(75)90026-6.

25. Dubois B, Burn D, Goetz C, et al. Diagnostic procedures for Parkinson's disease

dementia: Recommendations from the movement disorder society task force. Mov Disord.

2007;22(16):2314-2324; doi: 10.1002/mds.21844.

26. Emre M, Aarsland D, Brown R, et al. Clinical diagnostic criteria for dementia associated with Parkinson's disease. Mov Disord. 2007;22(12):1689-1707; doi: 10.1002/mds.21507.

27. Hsu YL, Chen CC, Lin YT, et al. Evaluation and Optimization of Sample Handling

Methods for Quantification of Short-Chain Fatty Acids in Human Fecal Samples by GC-MS.

J Proteome Res. 2019;18(5):1948-1957; doi: 10.1021/acs.jproteome.8b00536.

28. Liao HY, Wang CY, Lee CH, Kao HL, Wu WK, Kuo CH. Development of an Efficient

and Sensitive Chemical Derivatization-Based LC-MS/MS Method for Quantifying Gut

Microbiota-Derived Metabolites in Human Plasma and Its Application in Studying

Cardiovascular Disease. J Proteome Res. 2021 May 31. doi: 10.1021/acs.jproteome.1c00147.

29. Franzosa EA, McIver LJ, Rahnavard G, et al. Species-level functional profiling of metagenomes and metatranscriptomes. Nat Methods. 2018;15(11):962-968; doi:

10.1038/s41592-018-0176-y. 
30. Oksanen J, Blanchet FG, Friendly M, et al. Community Ecology Package. 2020.

Updated September 16, 2021. https://CRAN.R-project.org/package=vegan

31. Team RC. R: A language and environment for statistical computing. R Foundation for Statistical Computing, Vienna, Austria 2020.

32. Koh A, De Vadder F, Kovatcheva-Datchary P, Backhed F. From Dietary Fiber to Host Physiology: Short-Chain Fatty Acids as Key Bacterial Metabolites. Cell. 2016;165(6):13321345; doi: 10.1016/j.cell.2016.05.041.

33. Cilia R, Piatti M, Cereda E, et al. Does Gut Microbiota Influence the Course of Parkinson's Disease? A 3-Year Prospective Exploratory Study in de novo Patients. $J$ Parkinsons Dis. 2021;11(1):159-170; doi: 10.3233/JPD-202297.

34. Parada Venegas D, De la Fuente MK, Landskron G, et al. Short Chain Fatty Acids (SCFAs)-Mediated Gut Epithelial and Immune Regulation and Its Relevance for Inflammatory Bowel Diseases. Front Immunol. 2019 Mar 11;10:277; doi:

10.3389/fimmu.2019.00277.

35. Reichardt N, Duncan SH, Young P, et al. Phylogenetic distribution of three pathways for propionate production within the human gut microbiota. ISME J. 2014;8 (6):1323-1335; doi:

10.1038/ismej.2014.14.

36. Nishiwaki H, Hamaguchi T, Ito M, et al. Short-Chain Fatty Acid-Producing Gut Microbiota Is Decreased in Parkinson's Disease but Not in Rapid-Eye-Movement Sleep Behavior Disorder. mSystems. 2020;5(6):e00797-20; doi:10.1128/mSystems.00797-20

37. Kumari S, Goyal V, Kumaran SS, Dwivedi SN, Srivastava A, Jagannathan NR. 
Quantitative metabolomics of saliva using proton NMR spectroscopy in patients with

Parkinson's disease and healthy controls. Neurol Sci. 2020;41(5):1201-1210; doi:

10.1007/s10072-019-04143-4.

38. Kelly LP, Carvey PM, Keshavarzian A, et al. Progression of intestinal permeability changes and alpha-synuclein expression in a mouse model of Parkinson's disease. Mov

Disord. 2014;29(8):999-1009; doi: 10.1002/mds.25736.

39. Müller M, Hernández MAG, Goossens GH, et al. Circulating but not faecal short-chain

fatty acids are related to insulin sensitivity, lipolysis and GLP-1 concentrations in humans.

Sci Rep. 2019;9(1):12515; doi:10.1038/s41598-019-48775-0

40. Marizzoni M, Cattaneo A, Mirabelli P, et al. Short-Chain Fatty Acids and

Lipopolysaccharide as Mediators Between Gut Dysbiosis and Amyloid Pathology in

Alzheimer's Disease. J Alzheimers Dis. 2020;78(2):683-697; doi: 10.3233/JAD-200306.

41. Kim CH, Park J, Kim M. Gut microbiota-derived short-chain Fatty acids, T cells, and

inflammation. Immune Netw. 2014;14(6):277-288; doi: 10.4110/in.2014.14.6.277.

42. Markowiak-Kopec P, Slizewska K. The Effect of Probiotics on the Production of Short-

Chain Fatty Acids by Human Intestinal Microbiome. Nutrients. 2020;12(4):1107; doi:

10.3390/nu12041107.

43. Muniz Pedrogo DA, Chen J, Hillmann B, et al. An Increased Abundance of

Clostridiaceae Characterizes Arthritis in Inflammatory Bowel Disease and Rheumatoid

Arthritis: A Cross-sectional Study. Inflamm Bowel Dis. 2019;25(5):902-913; doi:

10.1093/ibd/izy318. 
44. Lindenberg F, Krych L, Fielden J, et al. Expression of immune regulatory genes

correlate with the abundance of specific Clostridiales and Verrucomicrobia species in the equine ileum and cecum. Sci Rep. 2019;9(1):12674; doi: 10.1038/s41598-019-49081-5.

45. Arumugam M, Raes J, Pelletier E, et al. Enterotypes of the human gut microbiome.

Nature. 2011;473(7346):174-180; doi: 10.1038/nature09944.

46. Lin HY, Tang CH, Chen YH, et al. Peptidoglycan enhances proinflammatory cytokine expression through the TLR2 receptor, MyD88, phosphatidylinositol 3-kinase/AKT and NFkappaB pathways in BV-2 microglia. Int Immunopharmacol. 2010;10(8):883-891;

doi:10.1016/j.intimp.2010.04.026

47. Bell SM, Burgess T, Lee J, Blackburn DJ, Allen SP, Mortiboys H. Peripheral Glycolysis

in Neurodegenerative Diseases. Int J Mol Sci. 2020;21(23):8924; doi:10.3390/ijms21238924

48. López KLR, Simpson JE, Watson LC, et al. TIGAR inclusion pathology is specific for

Lewy body diseases. Brain Res. 2019;1706:218-223; doi:10.1016/j.brainres.2018.09.032

49. Cassani E, Barichella M, Ferri V, et al. Dietary habits in Parkinson's disease: Adherence to Mediterranean diet. Parkinsonism Relat Disord. 2017;42:40-46; doi:

10.1016/j.parkreldis.2017.06.007.

50. Barichella M, Cereda E, Cassani E, et al. Dietary habits and neurological features of Parkinson's disease patients: Implications for practice. Clin Nutr. 2017;36(4):1054-1061; doi:10.1016/j.clnu.2016.06.020 
Table 1. Clinical characteristics and SCFAs levels in all participants.

\begin{tabular}{|c|c|c|c|}
\hline & $\begin{array}{l}\text { Healthy controls } \\
\quad(\mathrm{n}=85)\end{array}$ & $\begin{array}{l}\text { PD patients } \\
\quad(\mathrm{n}=96)\end{array}$ & $p$ value \\
\hline Age (years) & $64.8 \pm 8.2$ & $67.5 \pm 11.5$ & 0.075 \\
\hline Sex (male), n (\%) & $41(48.2)$ & $58(60.4)$ & 0.100 \\
\hline Height (cm) & $163.3 \pm 8.2$ & $162.8 \pm 8.0$ & 0.653 \\
\hline Weight $(\mathrm{Kg})$ & $64.6 \pm 7.5$ & $62.0 \pm 10.4$ & 0.155 \\
\hline BMI & $24.2 \pm 2.4$ & $23.4 \pm 3.4$ & 0.182 \\
\hline Education (years) & $13.1 \pm 2.2$ & $12.8 \pm 2.6$ & 0.463 \\
\hline Diabetes mellitus, n (\%) & $4(4.7)$ & $8(8.3)$ & 0.328 \\
\hline Hypertension, n (\%) & $18(21.2)$ & $26(27.1)$ & 0.355 \\
\hline Hyperlipidemia, n (\%) & $12(14.1)$ & $12(12.5)$ & 0.749 \\
\hline Disease duration (years) & N.A. & $6.1 \pm 5.1$ & \\
\hline Hoehn-and-Yahr stage (off) & N.A. & $2.3 \pm 1.2$ & \\
\hline Early stage, n (\%) & N.A. & $59(61.5)$ & \\
\hline Advanced stage, n (\%) & N.A. & $37(38.5)$ & \\
\hline Hoehn-and-Yahr stage (on) & N.A. & $1.8 \pm 0.9$ & \\
\hline MDS-UPDRS part III score (off) & N.A. & $27.5 \pm 20.1$ & \\
\hline MDS-UPDRS part III score (on) & N.A. & $17.3 \pm 12.5$ & \\
\hline Motor fluctuations, n (\%) & N.A. & $12(12.5)$ & \\
\hline Peak-dose dyskinesia, n (\%) & N.A. & $9(9.4)$ & \\
\hline MMSE score & N.A. & $25.7 \pm 6.5$ & \\
\hline LEDD & N.A. & $550 \pm 474$ & \\
\hline Usage of entacapone & N.A. & $12(12.5)$ & \\
\hline \multicolumn{4}{|l|}{ Fecal levels of SCFAs $(\mu \mathrm{mol} / \mathrm{g})$} \\
\hline Acetic acid (C2) & $386 \pm 204$ & $283 \pm 210$ & $0.004 * *$ \\
\hline Propionic acid (C3) & $145 \pm 101$ & $102 \pm 85$ & $0.006^{* *}$ \\
\hline Butyric acid (C4) & $69 \pm 44$ & $51 \pm 55$ & $0.003 * *$ \\
\hline Isobutyric acid (iC4) & $7.6 \pm 4.8$ & $7.8 \pm 5.5$ & 0.978 \\
\hline Valeric acid (C5) & $9.2 \pm 12.1$ & $9.0 \pm 9.6$ & 0.839 \\
\hline Isovaleric acid (iC5) & $4.7 \pm 3.3$ & $5.3 \pm 3.9$ & 0.479 \\
\hline \multicolumn{4}{|l|}{ Plasma levels of SCFAs $(\mu \mathrm{M})$} \\
\hline Acetic acid (C2) & $48 \pm 35$ & $45 \pm 39$ & 0.359 \\
\hline Propionic acid (C3) & $1.4 \pm 0.7$ & $1.8 \pm 1.0$ & $0.009^{*}$ \\
\hline Butyric acid (C4) & $3.4 \pm 2.3$ & $4.0 \pm 1.4$ & $0.004 * *$ \\
\hline Isobutyric acid (iC4) & $0.25 \pm 0.08$ & $0.29 \pm 0.12$ & 0.091 \\
\hline Valeric acid (C5) & $0.06 \pm 0.05$ & $0.10 \pm 0.13$ & $0.047^{*}$ \\
\hline Isovaleric acid (iC5) & $0.29 \pm 0.24$ & $0.32 \pm 0.25$ & 0.248 \\
\hline
\end{tabular}

BMI, body mass index; IBD, inflammatory bowel disease; IBS, irritable bowel syndrome;

LEDD, levodopa equivalent daily dose; MDS-UPDRS, Movement Disorder Society Unified 
PD Rating Scale; MMSE, Mini-Mental State Examination; N.A., not available; PD,

Parkinson's disease; SCFA, short chain fatty acid.

Variables are expressed as mean \pm standard deviation or number (percentage). ${ }^{*} p<0.05 ; * * p$ $<0.01$. 
1 Table 2. Receiver operating characteristic association statistics for predicting

2 development of PD.

\begin{tabular}{lcc}
\hline & AUC (95\% CI) & $p$ value \\
\hline Basic (age, sex) & $0.55(0.44-0.67)$ & 0.357 \\
$\begin{array}{l}\text { Basic + Fecal acetic acid, propionic acid, and } \\
\text { butyric acid }\end{array}$ & $0.65(0.56-0.75)$ & $0.004^{* *}$ \\
$\begin{array}{l}\text { Basic + Plasma propionic acid, butyric acid } \\
\text { and valeric acid }\end{array}$ & $0.67(0.57-0.78)$ & $0.003 * *$ \\
Full model $^{\mathrm{a}}$ & $0.72(0.63-0.81)$ & $<0.001 * *$ \\
\hline
\end{tabular}

3 The independent variables used in the models included continuous variables of age, fecal and

4 plasma levels of log transformed SCFAs levels and the categorical variable of sex.

$5 \quad{ }^{a}$ Full model indicated basic variables in addition to corresponding fecal and plasma targeted

6 biomarkers.

7 AUC, area under the curve; CI, confidence interval; SCFAs, short chain fatty acids.

$8 \quad *: p<0.05 ; * *: p<0.01$. 


\begin{tabular}{|c|c|c|c|c|c|c|}
\hline \multicolumn{4}{|c|}{ MDS UPDRS part III score } & \multicolumn{3}{|c|}{ MMSE score } \\
\hline & $\rho$ & Coefficient $(95 \% \mathrm{CI})$ & $p$ value & $P$ & Coefficient $(95 \% \mathrm{CI})$ & $p$ value \\
\hline \multicolumn{7}{|l|}{ Fecal level of SCFAs } \\
\hline Acetic acid (C2) & -0.37 & $-17.6(-31.0--4.14)$ & $0.012 *$ & 0.08 & $2.07(-1.33-5.46)$ & 0.226 \\
\hline Propionic acid (C3) & -0.32 & $-13.2(-25.4--0.93)$ & $0.036^{*}$ & 0.09 & $1.44(-1.44-4.32)$ & 0.319 \\
\hline Butyric acid (C4) & -0.40 & $-16.1(-26.9--5.28)$ & $0.004 * *$ & 0.17 & $2.95(0.60-5.31)$ & $0.015^{*}$ \\
\hline Isobutyric acid (iC4) & -0.17 & $-21.7(-36.2--7.25)$ & $0.004 * *$ & 0.08 & $-0.22(-4.01-3.57)$ & 0.909 \\
\hline Isovaleric acid (iC5) & -0.14 & $-14.2(-28.6-0.08)$ & 0.051 & 0.03 & $-1.10(-4.76-2.56)$ & 0.548 \\
\hline Valeric acid (C5) & -0.24 & $-6.94(-16.1-2.22)$ & 0.134 & -0.05 & $0.40(-1.78-2.57)$ & 0.714 \\
\hline \multicolumn{7}{|l|}{ Plasma level of SCFAs } \\
\hline Acetic acid (C2) & -0.18 & $-0.05(-19.1-18.9)$ & 0.996 & 0.05 & $4.50(-1.18-10.2)$ & 0.115 \\
\hline Propionic acid (C3) & 0.26 & $24.4(1.00-47.7)$ & $0.042 *$ & -0.11 & $-0.57(-8.85-7.71)$ & 0.887 \\
\hline Butyric acid (C4) & -0.01 & $16.9(-15.5-49.2)$ & 0.297 & -0.09 & $-11.7(-21.9--1.44)$ & $0.027^{*}$ \\
\hline Isobutyric acid (iC4) & 0.11 & $26.0(-5.27-57.3)$ & 0.100 & -0.41 & $-6.75(-17.7-4.22)$ & 0.216 \\
\hline Isovaleric acid (iC5) & -0.09 & $-6.33(-25.9-13.2)$ & 0.515 & -0.29 & $-3.54(-11.5-4.39)$ & 0.364 \\
\hline
\end{tabular}


1 Spearman's rank sum tests and multivariate linear regression models were applied to evaluate the correlations between SCFAs and disease

2 severity and duration. In the regression models of disease severity, the severity of motor function (MDS UPDRS part III motor score) or

3 cognitive performance (MMSE) was the dependent factor. The independent variables included log-transformed SCFAs, age, sex, disease

4 duration, LEDD and usage of entacapone. As for analyses of SCFAs and disease duration, disease duration was the dependent factor in the 5 regression model and independent factors included log transformed SCFAs, age and sex.

6 CI, confidence interval; LEDD, levodopa equivalent daily dose; MDS-UPDRS, Movement Disorder Society Unified PD Rating Scale; MMSE,

7 Mini-Mental State Examination; SCFA, short chain fatty acid.

$8 * p<0.05 ; * *<<0.01$ 


\title{
Neurology
}

\author{
Association of Fecal and Plasma Levels of Short-Chain Fatty Acids With Gut Microbiota \\ and Clinical Severity in Parkinson Disease Patients \\ Szu-Ju Chen, Chieh-Chang Chen, Hsin-Yu Liao, et al. \\ Neurology published online January 7, 2022 \\ DOI 10.1212/WNL.0000000000013225
}

\section{This information is current as of January 7, 2022}

Updated Information \&

Services

Citations

Subspecialty Collections

Permissions \& Licensing

Reprints including high resolution figures, can be found at: http://n.neurology.org/content/early/2022/01/07/WNL.0000000000013225.f ull

This article has been cited by 2 HighWire-hosted articles: http://n.neurology.org/content/early/2022/01/07/WNL.0000000000013225.f ull\#\#otherarticles

This article, along with others on similar topics, appears in the following collection(s):

Class III

http://n.neurology.org/cgi/collection/class_iii

Gastrointestinal

http://n.neurology.org/cgi/collection/gastrointestinal

Parkinson's disease/Parkinsonism

http://n.neurology.org/cgi/collection/parkinsons_disease_parkinsonism

Information about reproducing this article in parts (figures,tables) or in its entirety can be found online at:

http://www.neurology.org/about/about_the_journal\#permissions

Information about ordering reprints can be found online:

http://n.neurology.org/subscribers/advertise

Neurology ${ }^{\circledR}$ is the official journal of the American Academy of Neurology. Published continuously since 1951, it is now a weekly with 48 issues per year. Copyright Copyright (C) 2022 The Author(s). Published by Wolters

Kluwer Health, Inc. on behalf of the American Academy of Neurology.. All rights reserved. Print ISSN:

0028-3878. Online ISSN: 1526-632X.

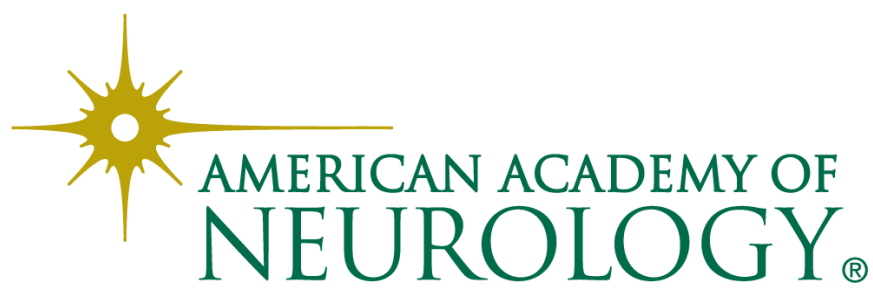

